\title{
An IL6-correlated signature in serous epithelial ovarian cancer associates with growth factor response
}

Patrizia Pinciroli ${ }^{1}$, Chiara Alberti ${ }^{1}$, Marialuisa Sensi ${ }^{2}$, Silvana Canevari ${ }^{1 *}$ and Antonella Tomassetti ${ }^{*}$

\begin{abstract}
Background: Epithelial ovarian cancer (EOC) is one of the most lethal gynecological cancers; the majority of EOC is the serous histotype and diagnosed at advanced stage. IL6 is the cytokine that has been found most frequently associated with carcinogenesis and progression of serous EOCs. IL6 is a growth-promoting and anti-apoptotic factor, and high plasma levels of IL6 in advanced stage EOCs correlate with poor prognosis. The objective of the present study was to identify IL6 co-regulated genes and gene network/s in EOCs.
\end{abstract}

Results: We applied bioinformatics tools on 7 publicly available data sets containing the gene expression profiles of 1262 EOC samples. By Pearson's correlation analysis we identified, in EOCs, an IL6-correlated gene signature containing 40 genes mainly associated with proliferation. 33 of 40 genes were also significantly correlated in low malignant potential (LMP) EOCs, while 7 genes, named C5AR1, FPR1, GOS2, IL8, KLF2, MMP19, and THBD were IL6-correlated only in advanced stage EOCs. Among the 40-gene signature EGFR ligand HBEGF, genes of the EGR family members and genes encoding for negative feedback regulators of growth factor signaling were included. The results obtained by Gene Set Enrichment and Ingenuity Pathway Analyses enabled the identification, respectively, of gene sets associated with 'early growth factor response' for the 40-gene signature, and a biological network related to 'thrombosis and cardiovascular disease' for the 7-gene signature. In agreement with these results, selected genes from the identified signatures were validated in vitro by real time RT-PCR in serous EOC cell lines upon stimulation with EGF.

Conclusions: Serous EOCs, independently of their aggressiveness, co-regulate IL6 expression together with that of genes associated to growth factor signaling, arguing for the hypothesis that common mechanism/s driven by EGFR ligands characterize both advanced-stage and LMP EOCs. Only advanced-stage EOCs appeared to be characterized by a scenario that involves genes which are so far associated with thrombosis and cardiovascular disease, thus suggesting that this pathway is implicated in the growth and/or spread of more aggressive tumors. We have discovered novel activated signaling pathways that drive the expression of IL6 and of co-regulated genes and are possibly involved in the pathobiology of EOCS.

Keywords: Epithelial ovarian cancer, IL6, Microarrays, Bioinformatics, Growth factor

\footnotetext{
*Correspondence: silvana.canevari@istitutotumori.mi.it; antonella.tomassetti@ istitutotumori.mi.it

'Unit of Molecular Therapies, Department of Experimental Oncology and Molecular Medicine, Fondazione IRCCS Istituto Nazionale dei Tumori, Milan, Italy

Full list of author information is available at the end of the article
} 


\section{Background}

Epithelial ovarian cancer (EOC) is the second most common and the most deadly malignancy of the female reproductive tract. Serous, endometrioid, clear-cell, and mucinous ovarian cancers are the four most common histotypes [1]. The majority of EOCs are diagnosed at stage III and IV when the tumor cells are spread in the peritoneum along with the presence of malignant ascites. The serous histotype accounts for about $80 \%$ of EOCs, and the majority show an inactivating mutation of the tumor suppressor gene TP53. Low malignant potential (LMP) serous EOCs are thought to arise by the transformation of tumors of borderline malignancy, and activating mutations in members of the RAS pathway (KRAS, BRAF, and ErbB2) are found in the majority of these tumors [2]. LMP EOCs show a relatively high growth capacity, are usually not invasive but resistant to conventional chemotherapy [1].

A number of studies suggest that factors related to the inflammation of the ovarian surface epithelium (OSE) such as ovulation, endometriosis, and pelvic inflammatory diseases are associated with an increased risk for EOC [3]. The most important hypothesis regarding EOC carcinogenesis is the ovulation theory, which relates the risk of ovarian cancer to incessant ovulation. Recently, it has been hypothesized that high grade serous, endometrioid and clear cell ovarian cancers arise from the fallopian tube epithelium and share a common pathogenic mechanism, i.e., iron-induced oxidative stress derived from retrograde menstruation [4]. Both the incessant ovulation and oxido-reductive fallopian tube epithelial damage hypotheses have provided evidence that inflammatory responses induced under physiological conditions may foster the development of EOC. In accordance with these hypotheses of ovarian tumorigenesis, a number of cyto/chemokines has been found at detectable levels in ascites from EOC patients [5]. Among those molecules, IL6 is the cytokine that has been most frequently associated with EOC carcinogenesis and progression [6]. Preclinical evidence has shown that IL6 enhances tumor cell survival and increases resistance to chemotherapy via JAK/STAT signaling in tumor cells [7] and IL6 receptor alpha trans-signaling on tumor endothelial cells $[8,9]$. In addition, IL6 has pro-angiogenic properties [7], regulating immune cell infiltration, a stromal reaction, and the tumor-promoting actions of Th17 lymphocytes [10]. In patients with advanced disease, high plasma levels of IL6 correlate with poor prognosis [11] and elevated levels are also present in malignant ascites [12]. Treatment of EOC cells with the anti-IL6 antibody (Ab) siltuximab has been shown to reduce constitutive cyto/chemokine production and inhibit IL6 signaling, tumor growth, the tumor-associated macrophage infiltrate, and angiogenesis in IL6-producing intraperitoneal ovarian cancer xenografts [13]. IL6 stimulates inflammatory cytokine production, tumor angiogenesis and the tumor macrophage infiltrate in ovarian cancer and these actions can also be inhibited by a neutralizing anti-IL6 Ab in clinical studies [14]. However, further knowledge on IL6-expressing EOCs is needed to select patients who are possibly responsive to IL6-dependent therapies.

We have recently found that IL6 can be co-expressed together with plasminogen activator inhibitor (PAI)-1, encoded by SERPINE1, in a subset of advanced stage serous EOCs due to the activation of the liganddependent EGFR/NFkB signaling cascade [15]. Ex vivo, using 23 EOCs from advanced-stage patients with malignant ascites at surgery, we observed co-expression of EGFR, IL6, and PAI-1 in 57\% of primary tumors and concomitant expression of both IL6 and PAI-1 in the corresponding ascites. Computational analysis on four publicly available data sets of EOC gene expression showed a correlation between the expression of the IL6 and SERPINE1 genes in advanced stage EOC patients, which in one case was associated with shorter progressionfree survival [15]. These results further highlight the involvement of IL6 in the progression of EOC.

Herein, to give further insight in the biology of IL6expressing serous EOC we utilized a bioinformatics approach, described in the flowchart of Figure 1, to identify IL6 co-regulated genes and signaling pathway/s in which they are involved. First, we identified a list of genes representing a molecular signature for both advancedstage and LMP serous EOC which recapitulate the socalled 'early growth factor response'. We also identified an IL6-correlated signature of seven genes involved in vascular thrombosis specific for advanced-stage serous EOCs.

\section{Results}

IL6 expression significantly correlates with a defined gene set in advanced stage serous EOCs

Pearson's correlation analysis of seven data sets containing the expression profiles of 1262 samples from serous EOCs (Table 1) was performed to identify genes whose expression was significantly correlated with IL6 expression in each data set. Correlation scores of each gene pair were computed using the $\mathrm{R}$ program essentially as described [16-18]. For genes represented by multiple probes in the same array format, the probe with the highest correlation to IL6 in the data set with the highest number of patients was chosen and considered for the other data sets when present (Additional file 1a). This analysis allowed the identification of genes whose expression positively correlated with IL6 along the seven data sets with a p-value $\leq 0.05$ and a Pearson's correlation coefficient $(r)$ exceeding 0.4 (Additional file 1b). A further analysis across the seven data sets yielded 40 


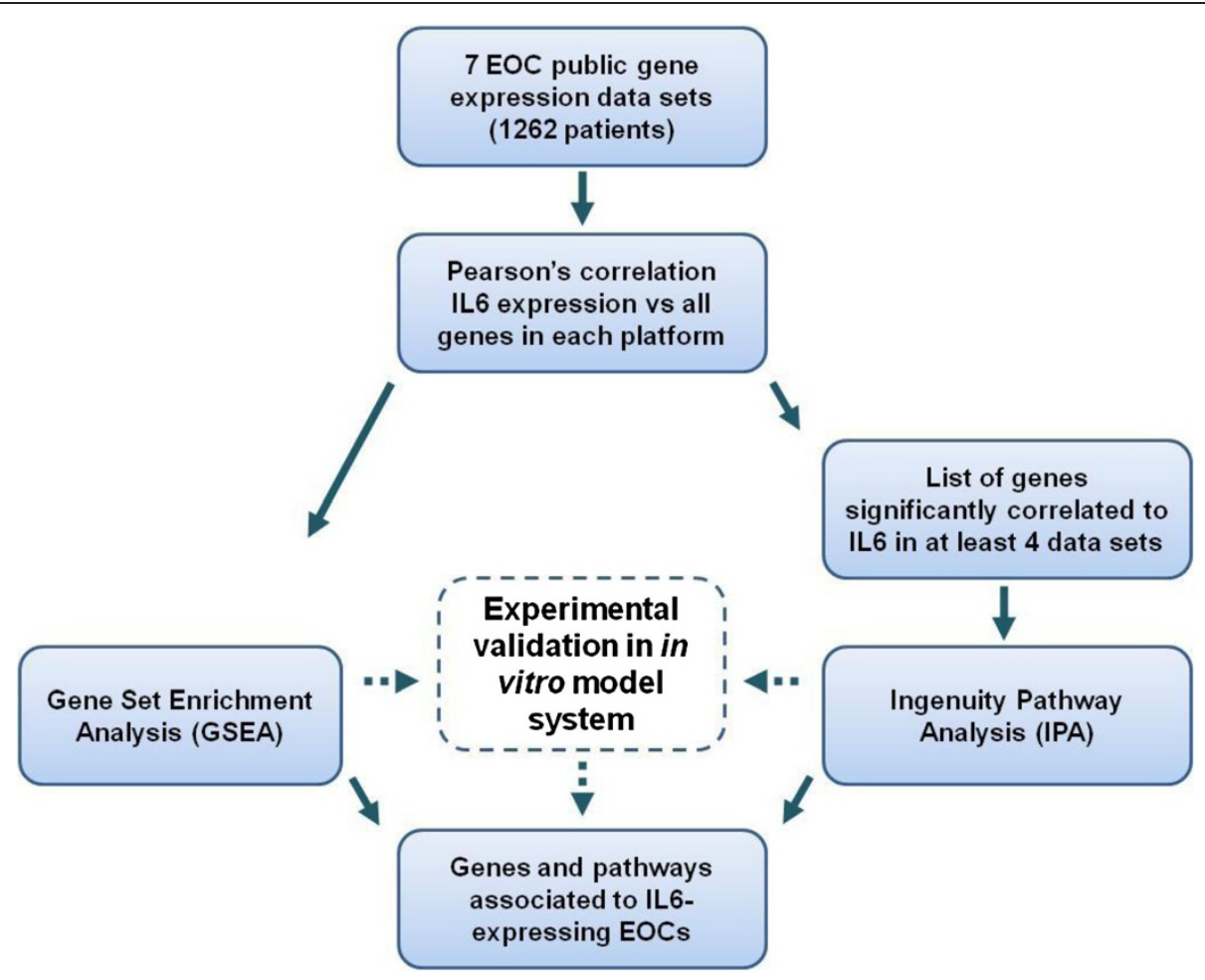

Figure 1 Flowchart describing the analysis workflow.

concordant correlated genes in at least four data sets (Additional file 1b and Figure 2). Of note, 38 of 40 genes were correlated in data set I (204 samples) obtained with an Affymetrix platform, and in data set VII (110 samples) obtained with an Agilent platform. Among the identified IL6-correlated genes CXCL2, HBEGF, SERPINE1, DUSP1, ZFP36, and IER3 were common to all data sets. The correlation between IL6 and HBEGF, an EGFR ligand, and SERPINE1, encoding PAI-1, is in agreement with our previously published results on co-expression of IL6 and PAI-1 in high grade EOCs due to EGFR activation [15]. The majority of genes are associated to the biological process 'proliferation' (50\%) (Table 2). Among the genes associated with proliferation, there were a number of growth factor early response genes (EGR1, EGR3, NR4A1,
FOSB, IER3). The IL6-correlated signature also included genes associated with 'inflammation' (20\%), and the remaining genes were associated with 'cell cycle and apoptosis,' 'metabolism' and 'migration and invasion'.

Thus, we identified a gene signature of IL6 correlated genes in serous EOC containing mainly proliferationassociated genes.

Advanced stage EOC-specific IL6-correlated gene signature functionally associated with control of cell morphology and cardiovascular disease

Next, to determine whether the identified gene signature was specific for advanced stage EOC or could also be associated with LMP EOC, Pearson's correlation analysis to IL6 was applied to gene expression data of LMP EOCs

Table 1 List of EOC data sets of gene expression analyzed in the present study

\begin{tabular}{|c|c|c|c|c|c|}
\hline \multirow{2}{*}{$\begin{array}{l}\text { Data } \\
\text { set ( ) }\end{array}$} & \multirow[t]{2}{*}{ Platform } & \multirow[t]{2}{*}{ Array } & \multirow{2}{*}{$\begin{array}{l}\text { No. of } \\
\text { probes }\end{array}$} & \multicolumn{2}{|c|}{ N. of serous EOC patients } \\
\hline & & & & Advanced stage & LMP \\
\hline I [19] & Affymetrix & HG-U133 Plus 2 & 54675 & 204 & 18 \\
\hline // [20] & Affymetrix & HG-U133 Plus 2 & 54675 & 60 & 30 \\
\hline III [21] & Affymetrix & HG-U133A & 22283 & 132 & 0 \\
\hline IV [22] & Affymetrix & HG-U133A & 22283 & 40 & 19 \\
\hline$V[23]$ & Affymetrix & HG-U133A & 22283 & 118 & 0 \\
\hline VI [24] & Affymetrix & HT_HG-U133A & 22277 & 598 & 0 \\
\hline VII [25] & Agilent & G4112A & 41000 & 110 & 0 \\
\hline
\end{tabular}




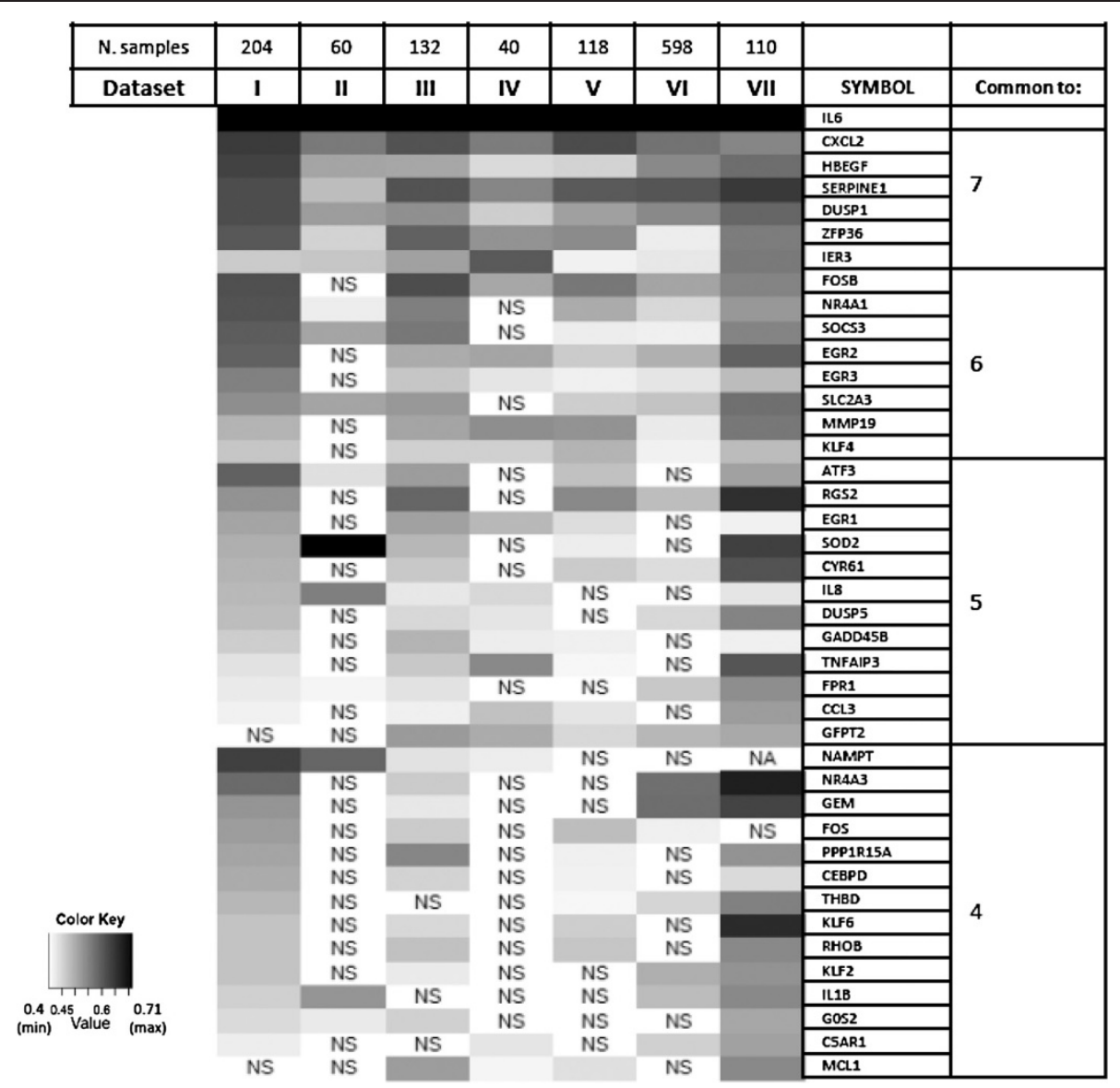

Figure 2 Heatmap of IL6-correlated genes. The heatmap of Pearson's correlation coefficient ( $r$ ) of the genes with IL6 was drawn by using $\mathrm{R}$ programming language. The $\mathrm{r}$ scores are represented in grayscale as reported in the color key. IL6 self-correlation was artificially set to the maximum score. Correlation score below 0.4 were considered not significant (NS). Genes not spotted on the array were defined NA (not available). The number of data sets in which the gene resulted significantly correlated with IL6 is reported on the right.

reported in data sets I, II, and IV (see Table 1). The density plot of IL6 intensities showed a similar trend of expression in advanced stage and LMP EOCs (Additional file 2). The data obtained comparing advanced stage EOCs and LMP EOCs were similar in the three data sets, and were more reliable (number of cases, genes identified, significance level) in data set I. Among the above identified advancedstage EOCs IL6-correlated genes, 33 were also significantly correlated in LMP EOCs, while 7 genes (C5AR1, FPR1, G0S2, IL8, KLF2, MMP19, and THBD) were specific for advanced-stages only (Additional file 3 ). Among these genes, IL8 has already been associated with aggressiveness and progression of malignant EOC [27], while the others have not previously been associated with EOC biology and clinical outcome.

To provide insight into the possible biological significance of the 40-gene signature, functional analysis of positively correlated genes (41, including IL6) was carried out by Ingenuity Pathway Analysis software (IPA) [28]. The top two functions (N1 and N2), associated with the highest score network, were 'Cell death, cellular function and maintenance, hematological system development and function' and 'Cell death, cellular development, cellular growth and proliferation' (Figure 3 and Additional file 4). When IPA analysis was performed on the seven-gene signature specific for advanced stage EOCs, the top function, associated with the highest score network, was 'Cell morphology, cell function, cardiovascular disease'. As shown in Figure 3 and listed in Additional file 4, all seven genes are included in this network (N3) together with genes already known to have a role in the progression of EOCs such as VEGF, the receptor tyrosine kinases EGFR and HER2, and the PI3K complex [1]. In addition to the input genes, it is noteworthy that IL6 is not present in the identified networks (Additional file 4), but when added manually to each network establishes a connection with some of the correlated genes (Figure 3).

The NFkB complex was included in networks N1 and N3 (Figure 3), highlighting its possible pivotal role in EOC progression. 
Table 2 Biological functions of the IL6-correlated genes

\begin{tabular}{|c|c|c|}
\hline Gene symbol & Name & Biological function $^{a}$ \\
\hline IL6 & interleukin-6 & Inflammation \\
\hline $\mathrm{CXCL2}$ & chemokine (C-X-C motif) ligand 2 & Inflammation \\
\hline HBEGF & heparin-binding epidermal growth factor & Proliferation \\
\hline SERPINE1 & plasminogen activator inhibitor 1 & Motility/Adhesion \\
\hline DUSP1 & dual specificity protein phosphatase 1 & Proliferation \\
\hline ZFP36 & tristetraprolin, zinc finger protein ZFP-36 & Proliferation \\
\hline IER3 & immediate early response 3 & Proliferation \\
\hline FOSB & $A P-1$, fos $B$ & Proliferation \\
\hline NR4A1 & TR3 orphan receptor, growth factor-inducible nuclear protein N10 & Proliferation \\
\hline SOCS3 & suppressor of cytokine signaling 3, cytokine-inducible $\mathrm{SH} 2$ protein 3 & Inflammation \\
\hline EGR2 & early growth response protein 2 & Proliferation \\
\hline EGR3 & early growth response protein 3 & Proliferation \\
\hline SLC2A3 & solute carrier family 2 (facilitated glucose transporter), member 3 & Metabolism \\
\hline MMP19 & matrix metalloproteinase-19 & Motility/Adhesion \\
\hline KLF4 & Krueppel-like factor 4 & Proliferation \\
\hline ATF3 & cyclic AMP-dependent transcription factor ATF-3 & Proliferation \\
\hline RGS2 & cell growth-inhibiting protein 31 , regulator of G-protein signaling 2 & Proliferation \\
\hline EGR1 & early growth response protein 1 & Proliferation \\
\hline SOD2 & manganese-containing superoxide dismutase, mitocondrial & Metabolism \\
\hline CYR61 & cysteine-rich, angiogenic inducer, 61 , IGF-binding protein 10 & Metabolism \\
\hline IL8 & interleukin 8 & Inflammation \\
\hline DUSP5 & dual specificity protein phosphatase 5 & Proliferation \\
\hline GADD45B & growth arrest and DNA damage-inducible protein GADD45 beta & Cell cycle control/Apoptosis \\
\hline TNFAIP3 & tumor necrosis factor, alpha-induced protein 3 & Inflammation \\
\hline FPR1 & formyl peptide receptor $1, \mathrm{~N}$-formylpeptide chemoattractant receptor & Inflammation \\
\hline CCL3 & chemokine (C-C motif) ligand 3 & Inflammation \\
\hline GFPT2 & hexosephosphate aminotransferase 2 & Metabolism \\
\hline NAMPT & nicotinamide phosphoribosyltransferase, pre-B-cell colony-enhancing factor 1 & Metabolism \\
\hline NR4A3 & Mitogen-induced nuclear orphan receptor, Nuclear hormone receptor NOR-1 & Proliferation \\
\hline GEM & RAS-like protein KIR, GTP-binding mitogen-induced T-cell protein & Proliferation \\
\hline FOS & AP-1, c-fos & Proliferation \\
\hline PPP1R15A & growth arrest and DNA-damage-inducible 34 & Cell cycle control/Apoptosis \\
\hline CEBPD & CCAAT/enhancer-binding protein delta, Nuclear factor NF-IL6-beta & Inflammation \\
\hline THBD & thrombomodulin & Motility/Adhesion \\
\hline KLF6 & Krueppel-like factor 6 & Proliferation \\
\hline $\mathrm{RHOB}$ & rho-related GTP-binding protein RhoB & Proliferation \\
\hline KLF2 & Krueppel-like factor 2 & Proliferation \\
\hline IL1B & interleukin 1, beta & Inflammation \\
\hline GOS2 & G0/G1 switch regulatory protein 2 & Cell cycle control/Apoptosis \\
\hline C5AR1 & complement component 5 receptor 1 & Motility/Adhesion \\
\hline MCL1 & bcl-2-like protein 3 & Cell cycle control/Apoptosis \\
\hline
\end{tabular}

${ }^{a}$ Biological functions were defined using GeneALaCart tool [26]. 


\section{N1}

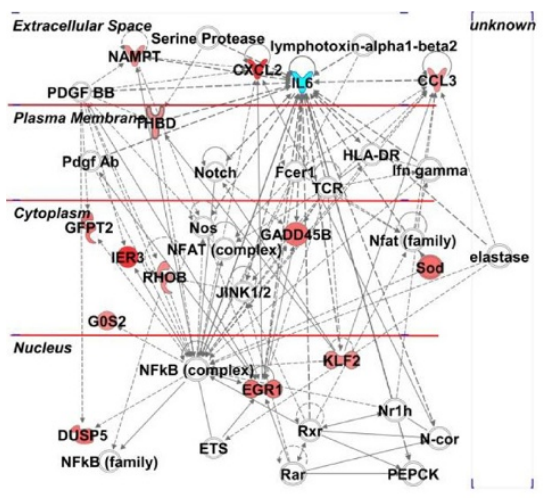

Cell Death, Cellular Function and Maintenance, Hematological System Development and Function

\section{N3}

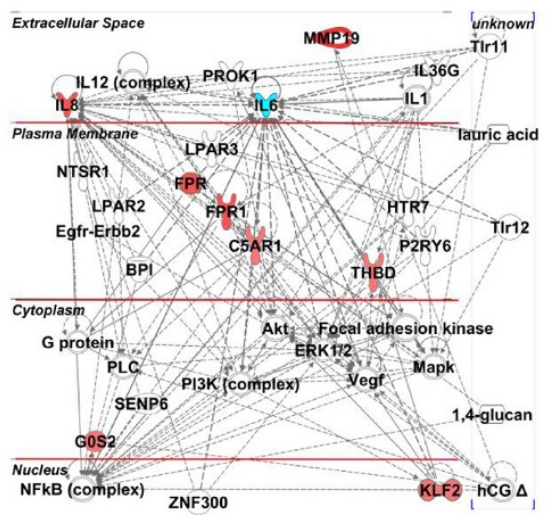

N2

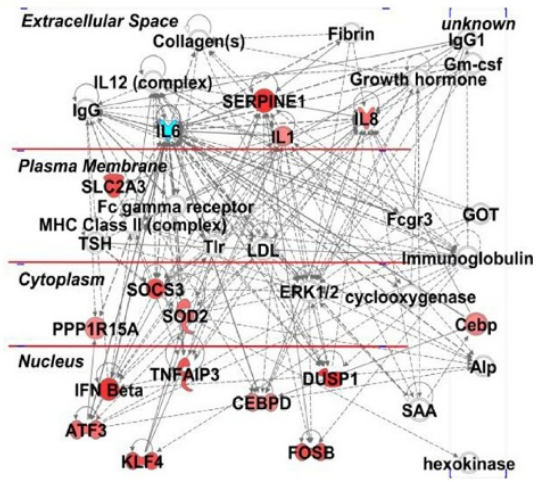

Cell Death, Cellular Development, Cellular Growth and Proliferation

Cell Morphology, Cellular Function and Maintenance, Cardiovascular Disease

Figure 3 Graphical representation of the top score networks identified by IPA. Molecular interactions between IL6-correlated genes in at least four data sets are reported. The top two networks (N1 and N2) were identified by loading all IL6-correlated genes in Fig. 1. Network 3 (N3) was identified by loading the 7 IL6-correlated genes specific for advanced stage EOCs. IL6 (highlighted in blue) was manually added to each network. IL6-correlated genes are highlighted in red and the intensity indicates the number of data sets where the gene is correlated. The name of the network is reported below the graph.

It thus appears that two signatures are related to IL6 in EOCs: a 33-gene signature common to advanced stage and LMP EOCs and associated to control of cell growth and death, while the 7-gene signature, associated only to advanced-stage EOCs likely presenting NFkB transcriptional activation, might be a determinant of tumor aggressiveness, and may be associated with a pathway regulating vascular thrombosis.

\section{An IL6-correlated gene set recapitulates the early growth} factor response

To give further insight in the biology of EOCs expressing IL6, a GSEA [29] analysis was performed for each data set listed in Table 1. By the "use a gene (IL6) as phenotype" analysis, GSEA first ranks the genes according to their correlation to IL6. It then determines whether a priori defined set of genes, in this instance those belonging to the $\mathrm{C} 2$ curated catalogue of functional gene sets, are randomly distributed throughout the gene list or primarily found at the top or bottom. Common significant gene sets obtained from GSEA analysis of the two largest data sets (I and VI) were selected and analyzed in the other datasets. This yielded 20 significantly enriched gene sets for all datasets. Normalized enrichment scores and FDR values in the different datasets are listed in Table 3. A literature search was conducted to identify signaling pathways previously implicated in the progression of EOCs and/or in epithelial- 
Table 3 Significant IL6 correlated gene sets identified by GSEA analysis.

\begin{tabular}{|c|c|c|c|c|c|c|c|c|c|c|c|c|c|c|}
\hline \multirow[b]{2}{*}{ GENESETS } & \multicolumn{2}{|r|}{ I } & \multicolumn{2}{|c|}{ II } & \multicolumn{2}{|c|}{ III } & \multicolumn{2}{|c|}{ IV } & \multicolumn{2}{|c|}{ v } & \multicolumn{2}{|c|}{ VI } & \multicolumn{2}{|c|}{ VII } \\
\hline & NES & $\begin{array}{l}\text { FDR } \\
\text { q-val }\end{array}$ & NES & $\begin{array}{l}\text { FDR } \\
\text { q-val }\end{array}$ & NES & $\begin{array}{l}\text { FDR } \\
\text { q-val }\end{array}$ & NES & $\begin{array}{l}\text { FDR } \\
\text { q-val }\end{array}$ & NES & $\begin{array}{l}\text { FDR } \\
\text { q-val }\end{array}$ & NES & $\begin{array}{l}\text { FDR } \\
\text { q-val }\end{array}$ & VES & $\begin{array}{l}\text { FDR } \\
\text { q-val }\end{array}$ \\
\hline AMIT_EGF_RESPONSE_120_HELA & 2,31 & 0,00 & 1,99 & 0,02 & 2,06 & 0,00 & 1,92 & 0,04 & 2,06 & 0,00 & 2,26 & 0,00 & 1,78 & 0,04 \\
\hline AMIT_EGF_RESPONSE_60_HELA & 2,35 & 0,00 & 1,96 & 0,02 & 2,08 & 0,00 & 2,15 & 0,01 & 2,21 & 0,00 & 2,31 & 0,00 & 1,89 & 0,03 \\
\hline BILD_HRAS_ONCOGENIC_SIGNATURE & 2,53 & 0,00 & 2,04 & 0,01 & 2,25 & 0,00 & 1,92 & 0,04 & 2,36 & 0,00 & 2,51 & 0,00 & 2,17 & 0,02 \\
\hline DAUER_STAT3_TARGETS_UP & 2,28 & 0,00 & 2,06 & 0,01 & 2,18 & 0,00 & 2,10 & 0,02 & 2,18 & 0,00 & 2,36 & 0,00 & 2,02 & 0,01 \\
\hline AZARD_RESPONSE_TO_UV_NHEH & 2,34 & 0,00 & 2,06 & 0,01 & 2,22 & 0,00 & 1,95 & 0,03 & 2,50 & 0,00 & 2,40 & 0,00 & 1,92 & 0,02 \\
\hline DIRMEIER_LMP1_RESPONSE_EARLY & 2,39 & 0,00 & 2,29 & 0,00 & 2,36 & 0,00 & 1,97 & 0,03 & 2,24 & 0,00 & 2,30 & 0,00 & 2,12 & 0,02 \\
\hline GERY_CEBP_TARGETS & 2,38 & 0,00 & 1,92 & 0,03 & 2,35 & 0,00 & 1,88 & 0,04 & 2,41 & 0,00 & 2,56 & 0,00 & 2,08 & 0,01 \\
\hline GRAHAM_CML_QUIESCENT_VS_NORMAL_DIVIDING_UP & 2,43 & 0,00 & 2,05 & 0,01 & 2,21 & 0,00 & 1,98 & 0,03 & 2,24 & 0,00 & 2,47 & 0,00 & 2,03 & 0,01 \\
\hline HALMOS_CEBPA_TARGETS_UP & 2,35 & 0,00 & 1,91 & 0,03 & 2,14 & 0,00 & 1,89 & 0,04 & 2,05 & 0,00 & 2,34 & 0,00 & 1,92 & 0,02 \\
\hline KIM_WT1_TARGETS_BHR_UP & 2,28 & 0,00 & 1,88 & 0,04 & 2,23 & 0,00 & 2,00 & 0,03 & 2,26 & 0,00 & 2,25 & 0,00 & 1,96 & 0,02 \\
\hline KIM_WT1_TARGETS_UP & 2,38 & 0,00 & 1,93 & 0,03 & 2,26 & 0,00 & 1,93 & 0,04 & 2,40 & 0,00 & 2,51 & 0,00 & 2,04 & 0,01 \\
\hline ARZEC_IL2_SIGNALING_UP & 2,34 & 0,00 & 2,16 & 0,01 & 2,03 & 0,01 & 1,60 & 0,15 & 1,88 & 0,02 & 2,28 & 0,00 & 2,03 & 0,01 \\
\hline NAGASHIMA_NRG1_SIGNALING_UP & 2,47 & 0,00 & 2,04 & 0,01 & 2,54 & 0,00 & 2,21 & 0,01 & 2,54 & 0,00 & 2,54 & 0,00 & 2,15 & 0,02 \\
\hline OSWALD_HEMATOPOIETIC_STEM_CELL_IN_COLLAGEN_ & 2,65 & 0,00 & 2,20 & 0,00 & 2,55 & 0,00 & 2,10 & 0,02 & 2,52 & 0,00 & 2,77 & 0,00 & 2,32 & 0,00 \\
\hline OSWALD_HEMATOPOIETIC_STEM_CELL_IN_COLLAGEN_ & 2,65 & 0,00 & 2,20 & 0,00 & 2,55 & 0,00 & 2,10 & 0,02 & 2,52 & 0,00 & 2,77 & 0,00 & 2,32 & 0,01 \\
\hline PICCALUGA_ANGIOIMMUNOBLASTIC_LYMPHOMA_DN & 2,47 & 0,00 & 1,94 & 0,03 & 2,12 & 0,00 & 1,95 & 0,03 & 2,28 & 0,00 & 2,25 & 0,00 & 2,00 & 0,02 \\
\hline SENESE_HDAC1_AND_HDAC2_TARGETS_UP & 2,41 & 0,00 & 1,88 & 0,04 & 2,04 & 0,01 & 2,00 & 0,03 & 2,07 & 0,00 & 2,62 & 0,00 & 2,16 & 0,02 \\
\hline SMIRNOV_CIRCULATING_ENDOTHELIOCYTES_IN_CANCE & 2,30 & 0,00 & 2,03 & 0,01 & 2,45 & 0,00 & 2,13 & 0,02 & 2,26 & 0,00 & 2,43 & 0,00 & 1,96 & 0,02 \\
\hline THEILGAARD_NEUTROPHIL_AT_SKIN_WOUND_UP & 2,45 & 0,00 & 2,02 & 0,01 & 2,24 & 0,00 & 1,96 & 0,03 & 2,16 & 0,00 & 2,26 & 0,00 & 1,91 & 0,02 \\
\hline VART_KSHV_INFECTION_ANGIOGENIC_MARKERS_UP & 2,36 & 0,00 & 1,93 & 0,03 & 2,20 & 0,00 & 1,78 & 0,07 & 2,19 & 0,00 & 2,63 & 0,00 & 1,99 & 0,02 \\
\hline ZHANG_RESPONSE_TO_IKK_INHIBITOR_AND_TNF_UP & 2,27 & 0,00 & 2,13 & 0,01 & 2,22 & 0,00 & 1,87 & 0,04 & 2,21 & 0,00 & 2,37 & 0,00 & 1,96 & 0,02 \\
\hline
\end{tabular}

NES normalized enrichment score, FDR false discovery rate.

derived malignancies. Among the most significant gene sets, the BILD_KRAS_ONCOGENIC_SIGNATURE [21] which includes genes whose expression is induced by the activation of H-RAS oncogene, was originally derived from the herein named data set III and can be considered a positive control. Three additional gene sets, AMIT_EGF_RESPONSE_60_HELA, AMIT_ EGF_RESPONSE_120_HELA [30] and NAGASHIMA_ NRG1_SIGNALING_UP [31], were considered possible candidates of signaling pathways associated with EOC, and are associated with 'growth factor response'. These gene sets comprise early response genes, i.e. the EGR family members, and the negative feedback regulators of the growth factor signaling, i.e. ZFP36 and KLF2. The fifth selected gene set, named KIM WT1_TARGET_UP in some ways also recapitulates the growth factor response, since among WT1 target genes the EGF family ligands EREG, AREG and HBEGF are included [32].

Furthermore, among the WT1 target genes SERPINE1 was also identified in the same study. Enrichment plots related to the above described gene-sets in data set I are shown in Figure 4. It is of note that IL6 is not included in the selected gene sets (Additional file 5) as well as other genes that are included in network 3 identified by analysis using IPA. Based on the results obtained by the abovedescribed computational analysis and on our recent demonstration that IL6 is up-modulated in EOC cells upon EGF stimulation in time-dependent manner [15], in vitro validation of 12 genes selected from the IL6-correlated gene sets was performed with real time RT-PCR using total RNA from EGF-stimulated serous EOC cell lines (Figure 5). The IL6 was up-modulated in all EOC cells analyzed upon EGF stimulation. Concordantly, $75 \%, 58 \%$, and $75 \%$ of the gene transcripts were up-modulated in IGROV1, OAW42, and SKOV3 cells, respectively (Figure 5). Among the correlated genes common to 7 data sets (see Figure 2), CXCL2, HBEGF, SERPINE1 and DUSP1 were increased in all three EOC cell lines analyzed. Additionally, NR4A1, a correlated gene in 6 data sets, was up-modulated upon EGF stimulation in all EOC cells. THBD and KLF2 transcripts, associated with 'Cardiovascular disease' by IPA analysis, were up-modulated in 2 of 3 EGF-stimulated EOC cells. In contrast, the MMP19 transcript, whose relevant protein is associated with invasion and tumor progression [33], was not up-modulated in EGF-stimulated 


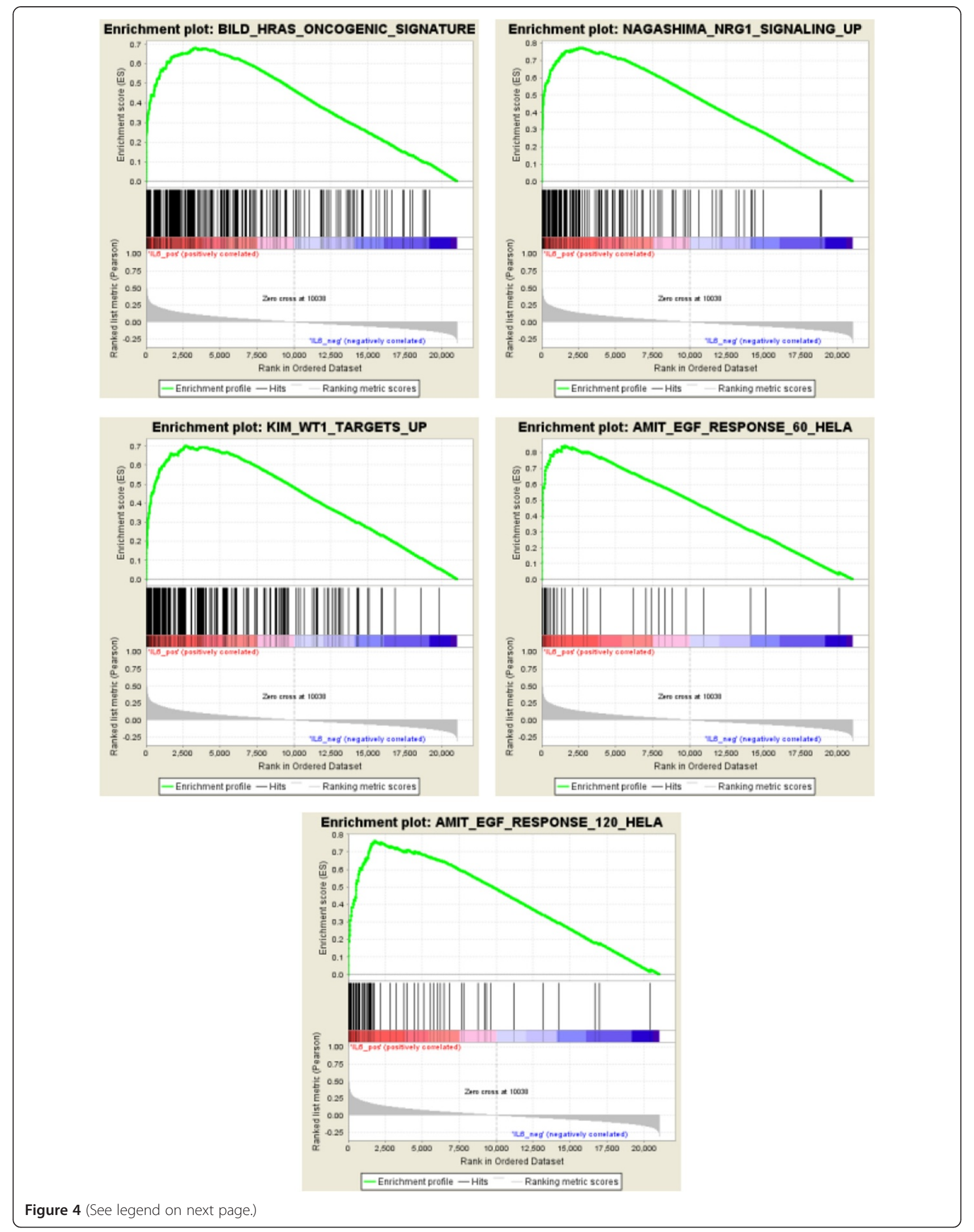


(See figure on previous page.)

Figure 4 GSEA enrichment plots for the five gene sets enriched in EOCs. On the top of each plot, the name of the gene set is reported. For each gene set, the enrichment plot was extracted from the GSEA output results and each gene set showed significant enrichment in IL6 expressing advanced stage EOC (FDR Q value = 0.0; Fig. 3). Genes with higher expression in IL6-positive tumors have higher enrichment scores, and are therefore plotted on the left side of the graph, whereas those with lower expression in IL6-positive tumors have lower enrichment scores and are plotted on the right side of the graph. The bottom portion of the plot shows the value of the ranking metric moving down the list of ranked genes. A positive ranking metric indicates that a gene is correlated with the IL6 positive phenotype. The results from dataset 1 are reported.

EOC cells. Interestingly, in non-transformed ovary cells, named IOSE- HTERT64 [34], although IL6 was slightly up-modulated by EGF stimulation, only $25 \%$ of the transcripts analyzed were up-modulated.

These data indicate that ligand-dependent EGFR activation in serous EOC cells induces the transcription of genes correlated with IL6 expression.

\section{Discussion}

Microarray technology has developed very rapidly, and it has become relatively easy to analyze the expression levels of thousands of genes within cancer cells. However, genes do not act in isolation, but each acts in complexes and builds networks and activated pathways that ultimately give rise to a specific cell phenotype. Thus,

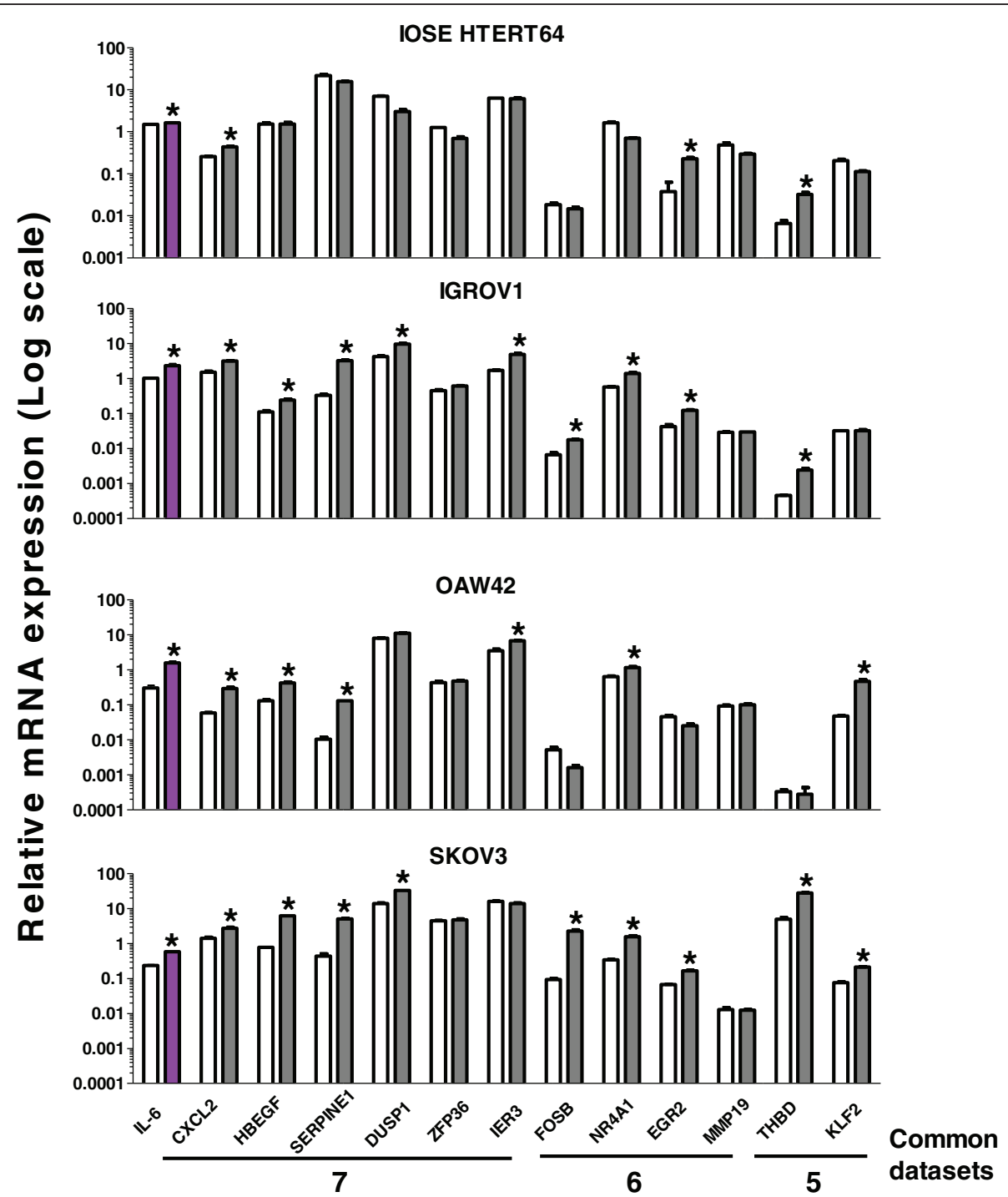

Figure 5 In vitro validation of selected IL6 correlated genes. Real time RT-PCR on selected IL6-correlated genes was performed using total RNA of starved EOC cell lines untreated (white bars) or treated (grey bars) for 4 hr (IGROV1, OAW42 and IOSE 64 hTERT) or 8 hr (SKOV3) with EGF $(20 \mathrm{ng} / \mathrm{ml})$. The number of data sets in which the gene resulted significantly correlated with IL6 is reported on the bottom. Data are mean values ( \pm SD) presented as relative expression normalized for GAPDH mRNA levels. Asterisks indicate significant positive variations (Student's $t$ test). 
the search of co-regulated genes applying bioinformatics approaches may spread light on the biology of a tumor and its development. Previously, by applying this kind of 'in silico' approach on gene expression profiles of ovarian and thyroid carcinomas [16,17] and melanomas [18], we have been able to identify novel signaling pathways activated in those tumors. The present study, by applying similar bioinformatics tools, highlights possible novel signaling pathways activated in IL6-expressing EOCs. Among those, growth factor-dependent signaling was also experimentally validated in vitro in selected cellular EOC models.

First of all, Pearson's correlation analysis allowed the identification of genes co-regulated with IL6 in aggressive EOC providing evidence that co-regulated genes can encode proteins involved in common signaling pathways. To identify IL6-coregulated genes we adopted thresholds which allowed to obtain a good balance among the statistical significance, the strength of the correlation and the biological reproducibility. Furthermore, we performed the analysis on 7 different data sets, containing the gene expression profiles of more than 1200 EOC samples, obtained on different array platforms, to increase the robustness on the bioinformatics results. We found a gene signature common to both advanced stage and LMP serous EOCs, and another 7-gene signature specific for advanced stage EOCs. The integration of the results obtained by IPA and GSEA, allowed us to determine that all EOCs, independently of their aggressiveness, co-regulate IL6 together with genes associated with cell growth and early growth factor response, arguing for the hypothesis of common mechanism/s of transformation. Only advanced-stage EOCs appeared to be characterized by a scenario that involves genes such as FPR1, KLF2 and THBD, to date associated with thrombosis and cardiovascular disease, thus suggesting that this pathway contributes to the growth and/or the spread of this type of tumor.

Our data indicate the existence of a biological interaction between IL6 expression and that of the coregulated genes as resulted upon IPA and GSEA analyses. On the other hand, since knockdown of IL- 6 by specific siRNA did not affect the amount of the transcripts analyzed (data not shown), the regulation of the expression of the identified genes appeared not directly dependent to that of IL6. Although the IL6 gene was not associated with the networks identified by IPA or with the gene sets selected by GSEA, these results are in agreement with our previous observations in a subset of advanced stage EOC where ligand-dependent EGFR activation induced NFkBdependent transcription of IL6 together with PAI-1, encoded by the SERPINE1 gene [15]. NFkB also emerged to be a possible transcriptional regulator of 13 out of 40 genes according to the reported informations [35], data which might further indicate that a growth factordependent NFkB signaling is activated in a subset of EOC. It is noteworthy that IL6 and 19 of the 40 correlated genes were found up-modulated upon $2 \mathrm{hr}$ serum stimulation of quiescent keratinocytes [36]. We can therefore argue that the activation of growth factor activated signaling can either directly or indirectly induce the expression of IL6 and genes which likely play a role in the growth of EOCs. Furthermore, this growth factor-induced signaling pathway induces positive regulators of cellular function that are in turn regulated by negative feedback regulators such as ZFP36 and KLF2 [30]. The HBEGF gene, encoding for an EGFR ligand, was also highly significantly correlated in all seven data sets analyzed, indicating the prevalence of ligand-dependent EGFR activation. The regulation of growth factor signaling pathways by negative feedback is a universal mechanism for limiting the duration and intensity of signaling output. While negative feedback is a key component of normal cellular signaling, its role in cancer cells is more complex. Indeed, the loss of some negative feedback regulators might contribute to tumor progression, but might also be expressed at considerably higher levels in oncogene-mutant tumors as observed in BRAFmutated melanomas [37]. Interestingly, the presence of a feedback negative mechanism has also been associated with greater efficacy of growth factor receptor-targeted therapy [38]. The fact that in EOC cells, with active EGFR/NFkB/IL6 signaling, EGFR-targeted therapy was more effective might be due to the up-regulation of feedback negative regulators of growth factor signaling [15]. Taken together, these data suggest that the IL6-associated signature might have a translational impact helping to select EOC patients who are likely responsive to EGFRtargeted therapy. Experiments are now ongoing to verify this hypothesis.

Nuclear expression of the Wilm's tumor suppressor is found in OSE cells and in the majority of serous EOCs [39]. However, the corresponding gene, named WT1, has been never associated with IL6 gene expression. WT1 is required for kidney development, and the report in which the relevant gene set has been derived particularly emphasized the finding that the genes encoding the EGF family ligands EREG, AREG, and HBEGF may be transcriptionally regulated by WT1, orchestrating a finetuning of the EGF signaling pathway [32]. Altogether these observations support that the EGF signaling pathway is pivotal in the biology of EOC.

The gene signature common to advanced stage serous and LMP EOCs is not unexpected if one considers the theory that LMP EOCs derive from serous low grade EOC with a borderline morphologic phenotype [40]. However, if this is the case, advanced-stage and LMP tumors might share common genetic alterations that induce aberrant growth. In addition, in vitro validation 
experiments performed on gene transcripts of nontransformed surface ovary cells argue for the notion that the signature associated with a growth factor response is not expressed and/or EGF-dependent in normal ovary cells.

The bioinformatics approach also produced hypothesisgenerating results. The association of the 7-gene signature with advance stage EOCs is novel. At present only angiogenesis-related genes and proteins, such as VEGF and its receptor, have a well documented role in EOC biology and are already well-exploited targets in the therapy of more aggressive EOCs [39]. Our findings open new questions on the role of genes associated with thrombosis and cardiovascular disease in the progression of EOCs. It has been recently hypothesized that low-dose aspirin as antithrombotic therapy may inhibit progression rather than the induction of EOC [41]. Indeed, aspirin and selective $\mathrm{COX}$ inhibitors could reduce progression not only by inhibiting prostaglandin production, thus reducing inflammation, but also by negatively modulating thrombosis-associated genes. Therefore, the inhibition of both pathways synergistically might be an interesting approach to block the growth and dissemination of advanced stage EOCs.

MMP19, a gene of the 7-gene signature specific for malignant EOCs and encoding the metallo-protease (MMP) 19 was present in network 3 of IPA analysis, but was not in any of the gene sets selected by GSEA analysis. MMPs are key molecules of tumor cell invasion, including EOCs [42] and, since the majority of samples were advanced stage EOCs, MMP19 could be a new player in the dissemination of these tumors and experiments are now ongoing to test its presence and role in advanced stage EOCs.

\section{Conclusions}

By applying a bioinformatics approach we identified genes co-regulated with IL6 expression in clinicallyrelevant subtypes of EOC, their interactions in networks and pathways as well as their functional association to growth factor response. IL6 gene expression together with that of the correlated gene signature could help identifying EOC patient's subgroups in which the identified signaling pathways might be biologically relevant during the progression of the disease and, in the long term, might represent new pharmacological targets.

\section{Methods}

\section{Computational analysis}

Seven EOC data sets, six arrayed on Affymetrix platforms and one on an Agilent platform, were analyzed (Table 1). Raw data of data sets I, II, and III [19-21] were downloaded from the NCBI Gene Expression Omnibus (GEO) repository (IDs GSE9891, GSE12172 and GSE3149, respectively) and those of data set VI were downloaded from the proprietary repository [43]. Data sets IV and V $[23,24]$ were downloaded from the Duke Institute website as suggested in the original publications. The raw data from Affymetrix were normalized through the RMA method using the Expression Console software developed by Affymetrix. Upon quality control, probes were annotated with the current annotation files (version 32) for the proper array format. Normalized data of data set VII [25], obtained on Agilent platform, were downloaded from GEO (ID GSE17260).

For each data set, the expression data from serous histotype cases were selected. Since in all but one (IV), data sets the percent of cases at early stage (I-II) ranged from 0 to $10 \%$, no stage selection was applied; in the case of data set IV, in which stage I and II represented $50 \%$ of case material, to avoid difficulty in comparison with the others, only advanced stages (III-IV) were selected. According to these selection criteria, we considered our overall case material to be composed of advanced stage EOC. Each data set was analyzed separately and the gene expression intensity of IL6, represented by a single probe in all the analyzed array formats, was correlated to the remaining probes across all EOCs samples in the array. The Pearson's correlation coefficients (r), p and FDR values were calculated using cor, cor.test and p.adjust (using the Benjamini \& Hochberg method) functions, respectively, from the Stats package in $\mathrm{R}$ programming language (version 2.12.0). For genes represented by multiple probes in the same array format, the probe with the highest correlation to IL6 in the data set with the highest number of patients was chosen and considered for the other data sets when present. Only genes exhibiting a $p$ value $\leq$ 0.05 and $r \geq 0.4$ in at least 4 of the 7 data sets were considered significant (Additional file 1). In three studies (I, II and IV), serous LMP EOCs were also profiled and their expression data analyzed as described above. Correlation values to IL6 corresponding only to the list of genes significant in advanced stage EOC were further considered (Additional file 1). IPA (Ingenuity Systems, 2012 release), a software leveraging a manually reviewed repository of biological interactions and functional annotations was used to analyze the signalling pathways, cellular location, function and, network connections of the identified genes [28].

Gene Set Enrichment Analysis (GSEA) [29], was used to find whether a set of genes defined based on prior biological knowledge (e.g., those in a common signaling pathway) shows statistically significant correlations with IL6. Briefly, for each of the seven EOCs datasets, through the "use a gene as phenotype" option, GSEA ranks the genes according to their correlation with IL6. This ranked lists is then interrogated against gene sets 
contained within the $\mathrm{C} 2$ curated gene sets (c2.all.v3.0. symbols.gmt), a collection of 2516 gene sets that are part of the Molecular Signatures Database (MSigDB) v3.0 $(12,13)$. The primary GSEA result is the enrichment score (ES), which reflects the degree to which a gene set is overrepresented at either the top or bottom of the ranked list of genes. To estimate the statistical significance of the ES, a nominal $\mathrm{p}$ value is calculated by permuting the genes 1,000 times. The ES score is normalized to account for the gene set sizes (NES). Gene sets associated to a false positive rate (FDR) of less than 0.25 were considered significant.

\section{Reagents}

Recombinant human EGF was from Peprotech. Taqman ${ }^{\circledR}$ Gene Expression Assays were from Applied Biosystems (Foster City, CA, USA).

\section{Ovarian cancer cell lines}

SKOV3, IGROV1 (serous histotype) cell lines were obtained from ATCC and maintained in RPMI 1640 medium (Sigma Aldrich) with $10 \%$ fetal calf serum (FCS) (Hyclone, Logan, UT) and $2 \mathrm{mmol} / \mathrm{L}$ glutamine, in a $5 \% \mathrm{CO}_{2}$ humidified atmosphere at $37^{\circ} \mathrm{C}$. OAW42 (serous histotype, kindly provided by Dr. A. Ullrich, Max Planck Institute of Biochemistry, Martinsried, Germany) cells were cultured in MEM (Sigma Aldrich) and supplemented as above. IOSE-64 hTERT cells were maintained and prepared as described [34]. All cell lines used in this study were subjected to short tandem repeat (STR) analysis and the profiles were compared to publically available databases to verify their authenticity. For the in vitro validation, a time course (up to $24 \mathrm{hr}$ ) with EGF stimulation was performed and IL6 expression was monitored by real timer RT-PCR in order to assess the shorter time necessary to detect IL6 up-modulation. Based on this method IGROV1, OAW42 and IOSE 64 hTERT were EGF stimulated for $4 \mathrm{hr}$ and and SKOV3 for $8 \mathrm{hr}$.

\section{RNA Extraction and real time RT-PCR}

Real time RT-PCR on selected IL6-correlated genes was performed on total RNA extracted from EOC cell lines stimulated for $4 \mathrm{hr}$ (IGROV1 and OAW42 cells) and $8 \mathrm{hr}$ (SKOV3 cells) with EGF $(20 \mathrm{ng} / \mathrm{ml})$. Total RNA from cell lines was extracted using a commercial kit (Amersham Bioscience-GE Healthcare). RT-PCR analysis was performed as described [17]. Human GAPD (GAPDH) Endogenous Control (VIC/MGB Probe) (RefSeq NM_002046.3) was used as housekeeping gene for normalization among samples. The Taqman Assays used for amplification were: Hs00174131_m1 for IL6; Hs00236966_m1 for CXCL2; Hs00181813_m1 for HBEGF; Hs01126604_m1 for SERPINE1; Hs00610256_g1 for DUSP1; Hs00185658_m1 for ZFP36; Hs00174674_m1 for IER3; Hs00171851_m1 for FOSB; Hs00374230_m1 for NR4A1; Hs00166165_m1 for EGR2; Hs00275699_ for MMP19; Hs00264920_s1 for THBD; Hs003604396_g1 for KLF2 (Applied Biosystems). Data analysis was performed by the Sequence Detection System (SDS) 2.2.2 software (Applied Biosystems).

\section{Additional files}

\begin{abstract}
Additional file 1: Table containing: a. Selected probe sets for each platform; b. IL6-correlated genes in serous high malignant EOCs.

Additional file 2: Figure reporting IL6 distribution (density plot) in the three data sets containing expression data of both advanced stage (204, 60 and 40 patients in data set I, II and IV, respectively) and LMP (18, 30 and 19 patients in data set I, II and IV, respectively) EOCs.

Additional file 3: Table reporting IL6-correlated genes in serous advanced stage and LMP EOCs from data set $\mathrm{I}$.

Additional file 4: Table reporting the networks identified by IPA software.

Additional file 5: Table reporting IL6-correlated genes included in each the gene sets selected by GSEA.
\end{abstract}

\section{Abbreviations}

EOC: Epithelial ovarian cancer; LMP: Low malignant potential; OSE: Ovarian surface epithelium; IPA: Ingenuity pathway analysis; GSEA: Gene set enrichment analysis.

\section{Competing interests}

The authors declare that they have no competing interests.

\section{Authors' contributions}

PP carried out bioinformatics and statistical analysis. CA carried out the in vitro biological validation. MS contributed to the design of the study. SC and AT, conceived the study, participated in its design and coordination, and drafted the manuscript. All authors read and approved the final manuscript.

\section{Acknowledgements}

We thank Dr. Patrick Moore for English editing of the manuscript. Financial Support: Italian Association for Cancer Research (IG10302 to SC and IG13055 to AT) and to Italian Ministry of Health ("Progetto Oncologico di Medicina Molecolare: i Tumori Femminili" to SC and "Progetto Integrato in Oncologia" to AT).

\section{Author details}

'Unit of Molecular Therapies, Department of Experimental Oncology and Molecular Medicine, Fondazione IRCCS Istituto Nazionale dei Tumori, Milan, Italy. ${ }^{2}$ Unit of Human Tumor Immunobiology, Department of Experimental Oncology and Molecular Medicine, Fondazione IRCCS Istituto Nazionale dei Tumori, Milan, Italy.

\section{Received: 31 October 2012 Accepted: 25 July 2013}

Published: 26 July 2013

\section{Reference}

1. Bast RC Jr, Hennessy B, Mills GB: The biology of ovarian cancer: new opportunities for translation. Nat Rev Cancer 2009, 9:415-428.

2. Berns EM, Bowtell DD: The changing view of high-grade serous ovarian cancer. Cancer Res 2012, 72:2701-2704.

3. Fleming JS, Beaugie CR, Haviv I, Chenevix-Trench G, Tan OL: Incessant ovulation, inflammation and epithelial ovarian carcinogenesis: revisiting old hypotheses. Mol Cell Endocrinol 2006, 247:4-21.

4. Vaughan S, Coward JI, Bast RC Jr, Berchuck A, Berek JS, Brenton JD, Coukos G, Crum CC, Drapkin R, Etemadmoghadam D, Friedlander M, Gabra H, Kaye SB, Lord CJ, Lengyel E, Levine DA, McNeish IA, Menon U, Mills GB, Nephew 
KP, Oza AM, Sood AK, Stronach EA, Walczak H, Bowtell DD, Balkwill FR: Rethinking ovarian cancer: recommendations for improving outcomes. Nat Rev Cancer 2011, 11:719-725.

5. Kulbe H, Thompson R, Wilson JL, Robinson S, Hagemann T, Fatah R, Gould D, Ayhan A, Balkwill F: The inflammatory cytokine tumor necrosis factoralpha generates an autocrine tumor-promoting network in epithelial ovarian cancer cells. Cancer Res 2007, 67:585-592.

6. Lo CW, Chen MW, Hsiao M, Wang S, Chen CA, Hsiao SM, Chang JS, Lai TC, Rose-John S, Kuo ML, Wei LH: IL-6 trans-signaling in formation and progression of malignant ascites in ovarian cancer. Cancer Res 2011, 71:424-434.

7. Wang Y, Niu XL, Qu Y, Wu J, Zhu YQ, Sun WJ, Li LZ: Autocrine production of interleukin- 6 confers cisplatin and paclitaxel resistance in ovarian cancer cells. Cancer Lett 2010, 295:110-123.

8. Yadav A, Kumar B, Datta J, Teknos TN, Kumar P: IL-6 promotes head and neck tumor metastasis by inducing epithelial-mesenchymal transition via the JAK-STAT3-SNAIL signaling pathway. Mol Cancer Res 2011, 9:1658-1667.

9. Nilsson MB, Langley RR, Fidler IJ: Interleukin-6, secreted by human ovarian carcinoma cells, is a potent proangiogenic cytokine. Cancer Res 2005, 65:10794-10800

10. Wang L, Yi T, Kortylewski M, Pardoll DM, Zeng D, Yu H: IL-17 can promote tumor growth through an IL-6-Stat3 signaling pathway. J Exp Med 2009, 206:1457-1464.

11. Plante M, Rubin SC, Wong GY, Federici MG, Finstad CL, Gastl GA: Interleukin-6 level in serum and ascites as a prognostic factor in patients with epithelial ovarian cancer. Cancer 1994, 73:1882-1888.

12. Lane D, Matte I, Rancourt C, Piche A: Prognostic significance of IL-6 and IL-8 ascites levels in ovarian cancer patients. BMC Cancer 2011, 11:210.

13. Guo Y, Nemeth J, O'Brien C, Susa M, Liu X, Zhang Z, Choy E, Mankin H, Hornicek F, Duan Z: Effects of siltuximab on the IL-6-induced signaling pathway in ovarian cancer. Clin Cancer Res 2010, 16:5759-5769.

14. Dijkgraaf EM, Welters MJ, Nortier JW, Van der Burg SH, Kroep JR: Interleukin-6/interleukin-6 receptor pathway as a new therapy target in epithelial ovarian cancer. Curr Pharm Des 2012, 18:3816-3827.

15. Alberti C, Pinciroli P, Valeri B, Ferri R, Ditto A, Umezawa K, Sensi M, Canevari $\mathrm{S}$, Tomassetti A: Ligand-dependent EGFR activation induces the coexpression of IL-6 and PAI-1 via the NFkB pathway in advanced-stage epithelial ovarian cancer. Oncogene 2012, 31:4139-4149.

16. Castellano G, Reid JF, Alberti P, Carcangiu ML, Tomassetti A, Canevari S: New potential ligand-receptor signaling loops in ovarian cancer identified in multiple gene expression studies. Cancer Res 2006, 66:10709-10719.

17. Degl'Innocenti D, Alberti C, Castellano G, Greco A, Miranda C, Pierotti MA, Seregni E, Borrello MG, Canevari S, Tomassetti A: Integrated ligandreceptor bioinformatic and in vitro functional analysis identifies active TGFA/EGFR signaling loop in papillary thyroid carcinomas. PLoS One 2010, 5:e12701.

18. Sensi M, Catani M, Castellano G, Nicolini G, Alciato F, Tragni G, De SG, Bersani I, Avanzi G, Tomassetti A, Canevari S, Anichini A: Human cutaneous melanomas lacking MITF and melanocyte differentiation antigens express a functional AxI receptor kinase. J Invest Dermatol 2011, 131:2448-2457.

19. Tothill RW, Tinker AV, George J, Brown R, Fox SB, Lade S, Johnson DS, Trivett MK, Etemadmoghadam D, Locandro B, Traficante N, Fereday S, Hung JA, Chiew YE, Haviv I, Gertig D, DeFazio A, Bowtell DD: Novel molecular subtypes of serous and endometrioid ovarian cancer linked to clinical outcome. Clin Cancer Res 2008, 14:5198-5208.

20. Anglesio MS, Arnold JM, George J, Tinker AV, Tothill R, Waddell N, Simms L, Locandro B, Fereday S, Traficante N, Russell P, Sharma R, Birrer MJ, DeFazio A, Chenevix-Trench G, Bowtell DD: Mutation of ERBB2 provides a novel alternative mechanism for the ubiquitous activation of RAS-MAPK in ovarian serous low malignant potential tumors. Mol Cancer Res 2008, 6:1678-1690.

21. Bild AH, Yao G, Chang JT, Wang Q, Potti A, Chasse D, Joshi MB, Harpole D, Lancaster JM, Berchuck A, Olson JA Jr, Marks JR, Dressman HK, West M, Nevins JR: Oncogenic pathway signatures in human cancers as a guide to targeted therapies. Nature 2006, 19;439:353-357.

22. Cancer Genome Atlas Research Network: Integrated genomic analyses of ovarian carcinoma. Nature 2011, 474:609-615.

23. Berchuck A, Iversen ES, Luo J, Clarke JP, Horne H, Levine DA, Boyd J, Alonso MA, Secord AA, Bernardini MQ, Barnett JC, Boren T, Murphy SK, Dressman HK, Marks JR, Lancaster JM: Microarray analysis of early stage serous ovarian cancers shows profiles predictive of favorable outcome. Clin Cancer Res 2009, 15:2448-2455.

24. Dressman HK, Berchuck A, Chan G, Zhai J, Bild A, Sayer R, Cragun J, Clarke J, Whitaker RS, Li L, Gray J, Marks J, Ginsburg GS, Potti A, West M, Nevins JR, Lancaster JM: An integrated genomic-based approach to individualized treatment of patients with advanced-stage ovarian cancer. J Clin Oncol 2007, 25:517-525.

25. Yoshihara K, Tajima A, Yahata T, Kodama S, Fujiwara H, Suzuki M, Onishi Y, Hatae M, Sueyoshi K, Fujiwara H, Kudo Y, Kotera K, Masuzaki H, Tashiro H, Katabuchi H, Inoue I, Tanaka K: Gene expression profile for predicting survival in advanced-stage serous ovarian cancer across two independent datasets. PLOS One 2010, 5:e9615.

26. The Genecards database. http://www.genecards.org. 2012.Ref Type: Generic.

27. Shahzad MM, Arevalo JM, Rmaiz-Pena GN, Lu C, Stone RL, Moreno-Smith M, Nishimura M, Lee JW, Jennings NB, Bottsford-Miller J, Vivas-Mejia P, Lutgendorf SK, Lopez-Berestein G, Bar-Eli M, Cole SW, Sood AK: Stress effects on FosB- and interleukin-8 (IL8)-driven ovarian cancer growth and metastasis. J Biol Chem 2010, 285:35462-35470.

28. The Ingenuity Pathway Analysis Software. 2012. http://www.ingenuity.com.

29. The Gene Set Enrichment Analysis software. 2012. http://www.broad.mit.edu/ gsea.

30. Amit I, Citri A, Shay T, Lu Y, Katz M, Zhang F, Tarcic G, Siwak D, Lahad J, Jacob-Hirsch J, Amariglio N, Vaisman N, Segal E, Rechavi G, Alon U, Mills GB, Domany $E$, Yarden $Y$ : A module of negative feedback regulators defines growth factor signaling. Nat Genet 2007, 39:503-512

31. Nagashima T, Shimodaira H, Ide K, Nakakuki T, Tani Y, Takahashi K, Yumoto N, Hatakeyama M: Quantitative transcriptional control of ErbB receptor signaling undergoes graded to biphasic response for cell differentiation. J Biol Chem 2007, 282:4045-4056.

32. Kim HS, Kim MS, Hancock AL, Harper JC, Park JY, Poy G, Perantoni AO, Cam M, Malik K, Lee SB: Identification of novel Wilms' tumor suppressor gene target genes implicated in kidney development. J Biol Chem 2007, 282:16278-16287.

33. Delassus GS, Cho H, Hoang S, Eliceiri GL: Many new down- and upregulatory signaling pathways, from known cancer progression suppressors to matrix metalloproteinases, differ widely in cells of various cancers. J Cell Physiol 2010, 224:549-558.

34. De Cecco L, Marchionni L, Gariboldi M, Reid JF, Lagonigro MS, Caramuta S, Ferrario C, Bussani E, Mezzanzanica D, Turatti F, Delia D, Daidone MG, Oggioni M, Bertuletti N, Ditto A, Raspagliesi F, Pilotti S, Pierotti MA, Canevari S, Schneider C: Gene expression profiling of advanced ovarian cancer: characterization of molecular signature involving the fibroblast growth factor 2. Oncogene 2004, 23:8171-8183.

35. NF-kB Target Genes. http://www.bu.edu/nf-kb/gene-resources/target-genes.

36. Qi L, Higgins SP, Lu Q, Samarakoon R, Wilkins-Port CE, Ye Q, Higgins CE, Staiano-Coico L, Higgins PJ: SERPINE1 (PAI-1) is a prominent member of the early G0 --> G1 transition "wound repair" transcriptome in p53 mutant human keratinocytes. J Invest Dermatol 2008, 128:749-753.

37. Pratilas CA, Taylor BS, Ye Q, Viale A, Sander C, Solit DB, Rosen N: (V600E) BRAF is associated with disabled feedback inhibition of RAF-MEK signaling and elevated transcriptional output of the pathway. Proc Natl Acad Sci USA 2009, 106:4519-4524.

38. Chandarlapaty S: Negative feedback and adaptive resistance to the targeted therapy of cancer. Cancer Discov 2012, 2:311-319.

39. Masoumi MS, Amini A, Morris DL, Pourgholami MH: Significance of vascular endothelial growth factor in growth and peritoneal dissemination of ovarian cancer. Cancer Metastasis Rev 2012, 31:143-162.

40. Kurman RJ, Shih I: The origin and pathogenesis of epithelial ovarian cancer: a proposed unifying theory. Am J Surg Pathol 2010, 34:433-443.

41. Lo-Ciganic WH, Zgibor JC, Bunker CH, Moysich KB, Edwards RP, Ness RB: Aspirin, nonaspirin nonsteroidal anti-inflammatory drugs, or acetaminophen and risk of ovarian cancer. Epidemiology 2012, 23:311-319.

42. Kenny HA, Lengyel E: MMP-2 functions as an early response protein in ovarian cancer metastasis. Cell Cycle 2009, 8:683-688.

43. The Cancer Genome Atlas database. [https://tcga-data.nci.nih.gov/] 2012.

\section{doi:10.1186/1471-2164-14-508}

Cite this article as: Pinciroli et al:: An IL6-correlated signature in serous epithelial ovarian cancer associates with growth factor response. BMC Genomics 2013 14:508. 